\title{
ATIVIDADE FISICA ADAPTADA PARA A PESSOA COM DEFICIÊNCIA: O CROSSFIT ADAPTADO PARA UM GRUPO COM CADEIRANTES E AMPUTADO
}

\author{
ACTIVIDAD FISICA ADAPTADA PARA LA PERSONA CON DISCAPACIDAD: EL \\ CROSSFIT ADAPTADO PARA PERSONAS CON SILLAS DE RUEDAS Y AMPUTADO
}

\author{
PHYSICAL ACTIVITY ADAPTED TO THE DISABLED PERSON: THE CROSSFIT \\ ADAPTED AS AN EXPERIENCE FOR PEOPLE WITH WHEELCHAIRS AND \\ AMPUTEES
}

\author{
Danielle da Silva Pinheiro WELLICHAN ${ }^{1}$ \\ Marcella Garcia Ferreira dos SANTOS ${ }^{2}$
}

RESUMO: De forma geral, a prática de atividade física traz inúmeros benefícios para a saúde. Para pessoas com deficiência, os benefícios são ainda maiores tendo em vista a necessidade de cada pessoa. Força, agilidade, equilíbrio, coordenação motora são alguns deles, além de aspectos psicológicos e sociais, como a melhora na autoestima, motivação, confiança, promoção da saúde e socialização. Cada vez mais, o esporte adaptado tem sido aceito e ampliado graças aos bons resultados alcançados e, nesse contexto, algumas modalidades representam boas opções para as pessoas com deficiência. Objetivando conhecer mais sobre a atividade e seus benefícios enquanto atividade física, constatou-se que o CrossFit Adaptado tem proporcionado experiências em vários aspectos, conforme as relatadas neste texto, por meio de entrevistas com um grupo praticante. Espera-se que essa modalidade consiga visibilidade e que novas unidades (da modalidade) sejam criadas pelo país a fim de estender a experiência para outras pessoas.

PALAVRAS-CHAVE: Atividade física. Deficiência física. Amputação. Promoção da saúde. Crossift Adaptado.

RESUMEN: La práctica de actividad física trae innumerables beneficios para la salud. Para las personas con discapacidad, los beneficios son aún mayores teniendo en cuenta la necesidad de cada persona en particular. La fuerza, agilidad, equilibrio, coordinación motora, son algunos de ellos, además de aspectos psicológicos y sociales, como la mejora en la autoestima, la motivación, la confianza, la promoción de la salud y la socialización. Cada vez más, el deporte adaptado ha sido aceptado y ampliado gracias a los buenos resultados alcanzados y en ese contexto, algunas modalidades han sido buenas opciones para las personas con discapacidad. Con el objetivo de conocer más sobre la actividad y sus beneficios como actividad física, se constató que el CrossFit Adaptado ha proporcionado experiencias en varios

\footnotetext{
${ }^{1}$ Universidade Estadual Paulista (UNESP), Marília - SP - Brasil. Doutoranda em Educação. Bibliotecária e Pedagoga, Especialista em Educação Infantil, Especial e Transtornos Globais do Desenvolvimento. Mestra em Ciência da Informação. Membro do Grupo de Pesquisa Deficiências Físicas e Sensoriais - DeFSen. ORCID: <http://orcid.org/0000-0002-6978-7361>.E-mail: dany_unesp@yahoo.com.br

2 Universidade Estadual Paulista (UNESP), Botucatu - SP - Brasil. Fisioterapeuta, Aprimoramento em Fisioterapia Geral. Analista Técnica Especializada - Fisioterapeuta no INSS, Bauru - SP - Brasil. ORCID: <http://orcid.org/0000-0002-6406-1107>.E-mail: marcella_mail@yahoo.com.br
} 
aspectos, conforme a las relatadas en este texto, a través de entrevistas con un grupo practicante. Se espera que esta modalidad consiga visibilidad y que nuevas unidades de la modalidad sean creadas por el país para extender la experiencia a otras personas.

PALABRAS CLAVE: Actividad física. Deficiencia física. Amputación. Promoción de la salud. Crossfit Adaptado.

ABSTRACT: The practice of physical activity generally brings numerous health benefits. For people with disabilities, the benefits are even greater in view of the need of each individual in particular. Strength, agility, balance, motor coordination, are some of them, as well as psychological and social aspects, such as improvement in self-esteem, motivation, confidence, health promotion and socialization. Increasingly, adapted sport has been accepted and expanded thanks to the good results achieved and, in this context, some modalities have been good options for people with disabilities. In order to know more about the activity and its benefits as a physical activity, it was verified that the CrossFit Adapted has provided experiences in several aspects, as reported in this text, through interviews with a practicing group. It is hoped that this modality will gain visibility and that new units of the modality will be created by the country to extend the experience to other people.

KEYWORDS: Physical activity. Physical Deficiency. Amputation. Health promotion. Crossfit Adapted.

\section{Introdução}

O movimento traz ao ser humano a expressão de seu pensar, agir e sentir, seja em pequenos gestos ou grandes movimentos. Na prática de atividades físicas, por exemplo, os benefícios da movimentação são notórios para o desenvolvimento motor, psicológico e social dos indivíduos.

Desde exercícios mais simples até os adaptados, as pessoas com deficiência encontram novas oportunidades de manter, desenvolver ou reabilitar sua saúde (seja no aspecto físico, psicológico ou social), o que representa uma melhoria em relação à mobilidade, equilíbrio e outros fatores, contribuindo diretamente para a qualidade de vida de pessoas em condições especiais, limitadoras ou restritivas (WELLICHAN; SANTOS, 2019). Além disso, o exercício físico pode intervir na redução da dor musculoesquelética e atuar na prevenção de deformidades, aumentando a resistência pulmonar e cardiovascular (RODRIGUES; MUSSI; ALMEIDA, 2014). Psicologicamente, a atividade física atua na melhoria da autoestima, do autoconceito, da imagem corporal e das funções cognitivas, diminuindo o estresse, a ansiedade e o consumo de medicamentos (MARTINS; RABELO, 2008). 
Após a Segunda Guerra Mundial, a atividade física passou a fazer parte do processo de reabilitação de soldados que voltavam das batalhas com deficiências e amputações. Desde então, o esporte adaptado mostra-se como um instrumento para a reabilitação, promoção de saúde, recuperação e socialização, além de favorecer a autonomia e independência para indivíduos que buscam desenvolver e aperfeiçoar habilidades e orientação espacial. E a autoestima torna-se um fator de grande importância nesse contexto, pois vai contribuir não só para o emocional como também para todos os momentos nos quais o indivíduo se encontrar entre sua limitação/restrição e suas reais possibilidades, que podem até ser desconhecidas por ele, ainda.

Conforme Rufini e Teixeira-Arroyo (2013) a deficiência física ou motora, é caracterizada por alterações físicas no corpo humano, com origens em problemas ortopédicos, neurológicos ou de má formação congênita, que ocasionam comprometimento no desenvolvimento ou limitações para as tarefas motoras. Embora existam os casos de origem na gestação ou nascimento, a estatística de lesões provocadas em decorrência de casos traumáticos com acidentes automobilísticos, acidentes de trabalho, armas de fogo, quedas, é de $80 \%$; e não traumáticas com origens tumorais, infecciosa, vascular ou degenerativa de 20\% (LIANZA, 2001).

Seja para buscar melhor condicionamento ou para fins de competição, os treinos de CrossFit Adaptado para pessoas com deficiência têm alcançado adeptos e surge no cenário esportivo como uma prática saudável em ascensão.

Motivados por fatores pessoais, situacionais, emocionais ou de comportamento, as pessoas com deficiência também buscam no esporte medidas terapêuticas (SAMULSKI, 2002; ESCARTI; CERVELLO, 1994), e assim pode ser considerada como um "mecanismo facilitador da inclusão na sociedade" (NOCE; SIMIM; MELO, 2009)

Diante dessa realidade, buscou-se junto a um grupo praticante dessa modalidade, localizado em uma cidade no interior do estado de São Paulo, o entendimento sobre essa atividade e seus benefícios enquanto atividade física. Por meio de relatos de experiências, constataram-se os resultados dessa prática que, segundo eles, contribui diretamente para melhores posicionamentos e, consequentemente, para a qualidade de vida de cada um.

\section{O Esporte Adaptado para pessoas com deficiência}

Como citado anteriormente neste texto, o esporte adaptado surgiu como uma possibilidade de inserção na sociedade para vítimas da segunda grande guerra. Nesse período, 
o neurologista alemão Ludwig Guttmann (considerado o precursor da reabilitação pelo esporte para pessoas com deficiência), a pedido do governo britânico, iniciou um trabalho no Centro Nacional de Lesionados Medulares do Hospital de Stoke Mandeville, que tratava de vítimas da guerra. A partir deste momento, surgiram duas correntes de pensamento sobre a atividade física adaptada.

A primeira, cujo enfoque era médico, foi apresentada pelo neurologista citado como um auxílio na reabilitação de seus pacientes para amenizar não só problemas psicológicos como também trazer o valor terapêutico e auxiliar na interação do paciente com a sociedade, ainda no período hospitalar.

A segunda corrente surgiu nos Estados Unidos e considerou o enfoque esportivo como uma forma de inserção social passível de competitividade e superação dos limites individuais, respondendo às quatro categorias de objetivos citadas por Wheeler et al. (1999): reabilitação, oportunidade social, recrutamento e continuidade no esporte. Assim, a reabilitação apoiada na prática de esportes proporcionou o surgimento de jogos para as pessoas com deficiência, cada vez mais frequentes ao longo da história (ARAUJO, 1997; VARELA, 1989; ALENCAR, 1997; BRAZUNA; MAUERBERG-DE CASTRO, 2001).

No Brasil, o marco do desenvolvimento esportivo adaptado é de 1958, com a fundação do Clube dos Paraplégicos em São Paulo e do Clube do Otimismo no Rio de Janeiro, que ofereciam programas denominados ginástica médica, com a finalidade de prevenir doenças utilizando exercícios corretivos e de prevenção (PEDRINELLI, 1994; ADAMS, 1985). “Assim, surge o reconhecimento do esporte como canal de socialização positiva ou de inclusão social[...]" (PADRÃO, 2017, p. 144)

$\mathrm{Na}$ atualidade, os jogos paraolímpicos representam um grande demonstrativo da capacidade de superação e reabilitação de pessoas com deficiência, e comprovam que a atividade física pode ser realizada por qualquer deficiência desde que acompanhada por profissionais especializados. Esse acompanhamento é necessário não só para prevenir lesões como também para descobrir potenciais a serem explorados.

\begin{abstract}
Valências físicas como equilíbrio, força, agilidade e velocidade devem ser trabalhadas, por meio de movimentos como: correr, saltar, lançar, trepar, transportar, rolar e rastejar. Adquiridas essas habilidades motoras, torna-se cada vez mais evidente uma grande vontade de conhecer e participar mais ativamente de algum tipo de desporto, enfatizando as partes funcionais de uma pessoa com deficiência (BARBOZA; DUTRA, 2017, p. 159).
\end{abstract}

Dentre as possibilidades esportivas, Melo e Lopez (2002) citam: arco e flecha, atletismo, basquetebol sobre rodas, bocha, ciclismo, equitação, esgrima, futebol, halterofilismo, iatismo, 
lawn bowls (semelhante a bocha), natação, racquetball (semelhante ao tênis de mesa), rugby em cadeira de rodas, tênis de mesa, tiro ao alvo e voleibol.

Diante do fato de que cada indivíduo possui uma limitação específica, não há limite ou opção mais indicada. Cada indivíduo precisa analisar e avaliar o quê ser feito e aquilo que atende melhor às suas necessidades e habilidades, o que amplia as opções citadas pelos autores citados. "A escassez de informação básica a respeito da população com deficiência tem sido uma grande barreira para o planejamento de ações capazes de promover sua inserção no meio social (SILVA; HENRIQUE; ROCHA, 2015, p.90).

Essa busca pela informação correta, a análise e a avaliação sobre as reais condições e potencialidades do indivíduo com deficiência precisam ser respeitadas e realizadas por profissionais conscientes, livres de qualquer preconceito a respeito da deficiência. Cabe, ainda, à sociedade em seus diversos segmentos compreender que cada caso é único e não pode ser tratado na forma generalizada, ou seja, quando possível, oferecer condições para que o próprio indivíduo demonstre o que pode realizar, sem julgá-lo ou classificá-lo com padrões préestabelecidos.

\section{Método}

Junto a um grupo de pessoas com deficiência, praticantes do Crossift Adaptado, buscaram-se relatos dessa experiência, e, para isto, foram respeitados os trâmites éticos necessários. Foi elaborado um roteiro como instrumento para a coleta dos dados e realizado um teste piloto para verificar se atendia às necessidades do estudo. Logo após as modificações necessárias, foram agendadas entrevistas antes do treino com quatro participantes que se interessaram em compartilhar sua experiência: três cadeirantes e um amputado, frequentadores de um box de CrossFit que também oferece treinos para pessoas sem deficiência.

Para compor o referencial teórico apresentado neste texto, buscaram-se em materiais impressos e eletrônicos subsídios para melhor descrever o contexto da atividade física e, posteriormente, associar aos relatos coletados. Optou-se por não estabelecer um período temporal, devido a importância de autores e obras já publicadas sobre o assunto.

As entrevistas foram transcritas, analisadas e, quando necessário, agrupadas para melhor exemplificar a fala dos participantes, que no decorrer no próximo item são identificados como P1, P2, P3 (cadeirantes) e P4 (amputado).

\section{Resultados e Discussão}


O CrossFit Adaptado é oferecido desde o ano de 2017 em um box que atende às pessoas com e sem deficiência. O coach (educador físico especializado e licenciado na modalidade) trouxe a versão adaptada para o local a fim de oferecer oportunidade para reabilitação, socialização e superação pessoal com eficiência. Os treinos são oferecidos gratuitamente em três dias na semana e acompanhados pelo coach e fisioterapeuta local.

Os participantes da modalidade adaptada compartilham os equipamentos existentes no box, e as adaptações, quando necessárias, são criadas no ato do exercício. O limite de cada participante é respeitado e acompanhado a fim de se evitar lesões e desconfortos.

Diferentemente da realidade das academias de ginástica, o local não possui espelhos e dispõe em geral de amplo espaço em função dos treinos. Os equipamentos (bicicletas, "remadores", "cavalos", caixas, pesos, cordas, pneus, bolas...) ficam em cantos específicos, permitindo livre circulação. Na parte interna, há rampas e escadas com corrimão que também são utilizadas para treinos, assim como área externa, pois apresenta algumas rampas nas calçadas que são aproveitadas nas corridas. As portas são largas e há vagas para estacionamento na frente do box.

O grupo da modalidade adaptada é pouco numeroso, e, embora compartilhem do espaço e dos equipamentos, possui treinos diferenciados. A única deficiência presente nos treinos é a física, o que não significa que outras deficiências não possam ser beneficiadas com a prática, apenas não houve procura.

Dos sete participantes informados sobre o presente estudo, quatro se interessaram em compartilhar seus relatos na experiência do CrossFit Adaptado: três cadeirantes (dois do gênero feminino e um do masculino) e um amputado (do gênero masculino), cujos diagnósticos de suas deficiências (todas motoras), faixa etária e escolaridade estão descritas no quadro seguinte.

Quadro 1 - Caracterização dos participantes

\begin{tabular}{|c|c|l|l|}
\hline PARTICIPANTE & IDADE & \multicolumn{1}{|c|}{ DIAGNÓSTICO } & \multicolumn{1}{c|}{ ESCOLARIDADE } \\
\hline P1 & 56 & Sequela de poliomielite & Ensino Superior em andamento \\
\hline P2 & 36 & Paraplegia adquirida - queda & Ensino Médio completo \\
\hline P3 & 38 & Paraplegia adquirida - sequela de cirurgia & Ensino Médio completo \\
\hline P4 & 34 & & Ensino Superior em andamento \\
\hline
\end{tabular}




\begin{tabular}{|l|l|l|l|}
\hline & $\begin{array}{l}\text { Amputação transtibial }- \text { acidente de } \\
\text { trabalho }\end{array}$ & \\
\hline
\end{tabular}

Fonte: Elaboração das autoras

Dos quatro praticantes do CrossFit adaptado, P4 está nos treinos há seis meses, P1, P2 e P3 há um mês e todos apontam mudanças significativas em relação aos condicionamentos e convivência, conforme descrito a seguir.

Para P1, P2 e P4, o CrossFit é uma das atividades físicas que incluíram em seus cotidianos: P1 pratica tênis de campo e basquetebol sob rodas; P2 pratica tênis de campo e natação; P4 praticava basquete e musculação, antes do acidente, agora está voltando às práticas esportivas. P3 não praticava nenhuma atividade esportiva, pois alegou que não tinha tempo em virtude das longas jornadas de trabalho. Após a sequela da cirurgia, encontrou no esporte adaptado motivação para se reabilitar: começou a praticar natação e tornou-se um atleta nessa modalidade.

Todos os praticantes citados neste artigo já se conheciam, e a decisão de frequentar o CrossFit surgiu da indicação de outros praticantes do box. Nenhum deles conhecia essa modalidade, cujas motivações individuais foram: a busca pela qualidade de vida e fortalecimento muscular (P1, P2 e P4), emagrecer (P2), curiosidade (P3) e melhora no condicionamento físico e mental (P4).

Questionados sobre as dificuldades iniciais encontradas nos treinos, foram citadas: falta de força (P1, P2 e P4), falta de equilíbrio, medo de cair e se machucar (P4). P3 afirmou que não teve nenhuma dificuldade.

Sobre as dificuldades encontradas nos treinos, os praticantes apontaram: "Tenho dificuldade com os pesos" (P1); "Levantar o próprio corpo na cadeira de rodas é difícil, faço no meu limite" (P2); “Agora já estou acostumado, não tenho mais dificuldade em realizar os exercícios. O medo de me machucar também passou, estou mais ciente do que posso e do que consigo fazer." (P4) afirmou. P3 manteve a resposta de não encontrar dificuldade nos treinos.

O coach prepara exercícios para todos, com as adaptações necessárias para cada um. Bicicleta, corrida, levantamento de peso, alongamento são exercícios comuns a todos e supervisionados pelos profissionais do local.

A atividade física já fazia parte da rotina de P4, mas em um ambiente de academia ou ao ar livre. O participante relatou que, embora tenha diminuído sua frequência no ano anterior ao acidente sofrido, devido ao excesso de trabalho, "Sempre se preocupou em praticar atividades físicas para cuidar da saúde". E após o acidente, quando começou sua reabilitação, 
foi orientado também pela equipe médica sobre a contribuição da prática esportiva para sua condição atual.

Entre as diferenças em treinos no CrossFit ou em academias, as respostas foram variadas e apontam interessantes indicativos a respeito dos praticantes e seus motivos particulares, conforme descrito a seguir:

É mais empolgante. Os treinos são sempre diferentes e a segurança que o professor [coach] nos transmite ajuda bastante (P1)

A ausência daquele ambiente com espelhos nos dá mais ânimo para fazer exercícios (P2).

Um motiva o outro, é bem animado (P3)

É um ambiente que motiva a superação, os treinos são desafiadores, e aí acreditamos mesmo que podemos fazer qualquer coisa (P3)

Em uma academia o treino é solitário, no Cross ele é mais agitado e animado. Sem contar que a gente se diverte, damos risada de nós mesmos, porque as vezes a gente pensa que não consegue fazer e aí vai lá e faz! (P4).

Percebe-se que a variedade dos treinos é benéfica para os praticantes, não só na motivação quanto na proposta de acreditar no que podem fazer. Além disso, a questão de superar as próprias limitações ou receios é fator de motivação para cada um. Na resposta de P4 fica claro o quanto a convivência (pessoas com e sem deficiência) auxilia no processo de inclusão e da reabilitação propriamente dita.

Essa convivência citada por P4 é uma aliada importante no processo de inclusão, pois, infelizmente, “[...] existe a barreira que não é física, mas está ali. Essa barreira está escondida nas palavras, na forma de olhar, na forma de tratar" (BARBOZA; DUTRA, 2017, p. 160). E assim, não há como ou porquê a deficiência física passar despercebida, e seus sinais estão na aparência, tamanho e/ou funcionalidade denunciando a diferença (MARTINS; BARSAGLINI, 2011).

Essas marcas corporais podem afetar e deteriorar a identidade da pessoa com deficiência e, consequentemente, contribuir para o seu isolamento (GOFFMAN, 1988). Existindo essa possibilidade (de isolar-se), o "ser e sentir-se deficiente" exige que não só a pessoa com deficiência, mas todos a sua volta estejam dispostos a buscar condições para que o ideal da inclusão aconteça. No momento em que a pessoa com deficiência rompe seus limites e descobre suas potencialidades ao realizar sua rotina com independência e autonomia, ela resgata sua dignidade (MARTINS; BARSAGLINI, 2011).

A acessibilidade também interfere nesse resgate, pois pode favorecer ou comprometer a independência e a autonomia de uma pessoa que apresente deficiência ou alguma limitação, 
seja ela temporária ou permanente. E sobre esse assunto, nenhum dos participantes mencionou qualquer dificuldade no ambiente de treino.

Me sinto super acolhido porque não preciso que ninguém faça nada por mim, faço tudo o que preciso e quero fazer sozinho. (P3)

Não vejo dificuldade aqui, nem no espaço e nem com a gente mesmo, ninguém fica olhando ou julgando, a gente é a gente e divide o mesmo espaço. (P4)

Ao término do treino, os praticantes afirmam que se sentem "Com muita disposição" (P1 e P2), "Exausto, mas satisfeito com meu rendimento" (P3), "Cansado, mas muito bem mental e fisicamente" (P4).

Sobre os resultados percebidos desde o início dos treinos, as respostas foram variadas: "Percebi uma disposição maior, mas ainda espero mais resultados" (P1), "Percebi que estou com mais disposição nas atividades diárias" (P2), "Percebo que tem melhorado minha força muscular, o que me ajuda na natação" (P3) e "Desde que comecei, percebi melhora na minha respiração, ganhei força muscular o que é muito importante para minha protetização e minha autoestima melhorou bastante" (P4).

Pelas transcrições das falas dos participantes, percebe-se que, embora a atividade física já faça parte de suas rotinas, o CrossFit trouxe força muscular, o que tem favorecido outras atividades já praticadas, no caso de P1 e P2. Para P3, que aderiu às atividades após a cirurgia, a prática faz parte da descoberta das habilidades que antes não imaginava ter.

\begin{abstract}
Antes, eu só pensava em trabalhar, não fazia exercícios porque não tinha tempo mesmo. Trabalhava muito. Até achava interessante, mas nunca parei para fazer, hoje é diferente, se quero ter qualidade de vida preciso buscar. O fato de estar numa cadeira de rodas não me impede de fazer o que quero ou preciso (P3).
\end{abstract}

Anteriormente, P1, P2 e P3 identificaram a falta de força como uma dificuldade encontrada nos treinos; posteriormente, os participantes P3 e P4 voltaram a mencionar a questão da força, agora identificada em suas ações. De fato, a força torna-se uma exigência para a aquisição de melhor autonomia, equilíbrio, locomoção, movimentação e postura, seja para um cadeirante ou para um amputado.

Para o cadeirante, atividades que aumentem os níveis de força são facilitadoras em situações de transferência da cadeira para outro mobiliário ou local, por exemplo, uma cadeira, um carro, ou qualquer outro movimento. A resistência cardiovascular também precisa ser desenvolvida, pois o simples deslocamento pode causar fadiga e comprometer as atividades de vida diária (AVD). 
Embora o nível da amputação seja um fator diferencial e associado ao bom estado do membro residual (coto), para o amputado, o fortalecimento muscular, a descarga e a sobrecarga de peso, os treinos de marcha contribuirão para a protetização, que só será possível se houver condições musculares e funcionais na parte que irá receber a prótese. Dessa forma, voltar à vida diária vai requerer mais do que força de vontade, exigindo condições anatômicas, funcionais, além das articulações, funções cardiorrespiratórias e da qualidade da prótese (MATSUMURA; RESENDE; CHAMLIAN, 2013).

Após a amputação a gente fica em dúvida se vai conseguir fazer coisas simples, como sentar no chão ou se manter equilibrado. Quando comecei o Cross estava com muletas e tinha muitas dúvidas sobre o que conseguiria fazer. Hoje estou com a prótese e consigo fazer muitas coisas até sem ela porque meu equilíbrio e minha força muscular estão sendo trabalhadas e percebo isso a cada treino (P4).

Nessa retomada da vida diária, causada pela deficiência adquirida (os quatro participantes deste estudo são casos adquiridos), não são só as mudanças físicas que se tornam percebíveis ou visíveis: atitudes, opiniões, comportamentos, condutas se tornam até evidentes na medida em que acontecem. Nesse sentido, se antes a atividade física não fazia parte da vida de P3, após adquirir a deficiência, foi a motivação necessária para seu desenvolvimento. Para P4, a atividade física trouxe mais segurança para sua reabilitação física e emocional.

Os participantes deste estudo recomendam a pratica do CrossFit Adaptado, e, embora não tenham despertado o interesse pelas competições nessa prática, não descartam a possibilidade futura.

No momento não tenho interesse nas competições pelo CrossFit, mas acredito que possam ser bem gratificantes pessoalmente. (P3)

Olha, se alguém quiser me patrocinar e eu tiver condições de tentar, claro que me interesso. Quero agora descobrir meus limites e me superar cada vez mais. Isso me fortalece e me dá qualidade de vida. (P4)

Diante do exposto e proposto pelo presente estudo, a atividade física oferece possibilidades comprovadas de recuperação, superação e reabilitação. Pelas falas dos participantes, ficam evidentes os efeitos dessa prática na promoção da imagem mais segura e confiante, na obtenção de força muscular, dentre outros pontos necessários em todos os casos citados.

Embora o CrossFit esteja em crescimento no território nacional brasileiro, ainda precisa ser mais divulgado, principalmente nas modalidades adaptadas. Assim, outras pessoas com 
deficiência terão oportunidade de conhecer e participar, pois os benefícios advindos precisam ser compartilhados e vivenciados.

Diante de todo o exposto, Oliveira, Gonçalves e Seabra Jr. (2017, p.106) afirmam:

\begin{abstract}
Atualmente a inclusão é muito discutida, principalmente no âmbito escolar, porém, é importante salientar que uma inclusão efetiva na sociedade (por meio do esporte), e na escola (nas aulas de educação física), deve oferecer a prática esportiva as pessoas com deficiência, e quando necessário realizar adaptações práticas ou no ensino para que o esporte seja efetivamente praticado, ocasionando assim, uma equiparação de oportunidades.
\end{abstract}

\title{
Considerações Finais
}

O CrossFit Adaptado mostrou-se uma atividade física que pode trazer benefícios sob diversos aspectos para as pessoas com deficiência. Independentemente de suas particularidades, a atividade (ou treino), trouxe aos participantes deste estudo possibilidades importantes não só para a qualidade de vida, mas para a inclusão deles em um ambiente acessível e favorável à promoção da saúde.

Com objetivos diferentes, cada participante demonstrou que o se sentir com deficiência pode ou não coincidir com o fato de ser reconhecido como tal. Na deficiência, as limitações e as possibilidades podem depender da pessoa envolvida, dessa forma, avaliações são de extrema necessidade para constatar ou não restrições, limites pessoais e oportunidades para serem exploradas. A pessoa com deficiência, principalmente na deficiência adquirida, não deve ser tratada com padrões pré-estabelecidos, pois cada sujeito pode ter um potencial diferente do outro e não raramente ele também pode desconhecer seu potencial, por isso, a questão da oportunidade se torna tão importante.

A qualidade de vida alcançada pela pratica da atividade física torna-se evidente a partir do momento em que resultados são percebidos no cotidiano das pessoas, em especial daquelas que necessitam de independência e autonomia como uma forma de sentir-se parte da sociedade.

O número reduzido de participantes desta pesquisa não pode ser considerado como limitante para a análise dos dados coletados, tendo em vista a importância das falas de cada um.

Espera-se que modalidades como a do CrossFit Adaptado sejam mais frequentes e estejam disponíveis em mais localidades para que outras pessoas com deficiência tenham a oportunidade de usufruir dessa atividade, seja por lazer ou como parte de sua reabilitação. 


\section{REFERÊNCIAS}

ADAMS, R. C., et al. Jogos, esportes e exercícios para o deficiente físico. São Paulo: Manole, 1985.

ALENCAR, B. Paraolimpíada: o Brasil no pódio. Rio de Janeiro [s.n.], 1997.

ARAÚJO, P. F. de. Desporto adaptado no Brasil: origem, institucionalização e atualidade. 1997. 140f. Tese (Doutorado em Educação Física). Universidade Estadual de Campinas, SP. 1997.

BARBOZA, L. P.; DUTRA, F. B. S. O resgate da autoestima de pessoas com deficiência através da prática do Rugby em cadeira de rodas. In: DUTRA, F. B. S.; SANTOS, C. R. V.; DI BLASI, F. Aspectos da deficiência: educação, esporte e qualidade de vida. Curitiba: Appris, p. 157-168, 2017.

BRAZUNA, M. R.; MAUERBERG-DE CASTRO, E. A trajetória do atleta portador de deficiência física no esporte adaptado de rendimento. Uma revisão de literatura. Motriz, v. 7, n. 2, p. 115-123, jul./dez. 2001.

ESCARTÍ, A.; CERVELLÓ, E. La motivación en el deporte. En: ISABEL, 1994.

GOFFMAN, E. Estigma: notas sobre a manipulação da identidade deteriorada. Rio de Janeiro: Guanabara Koogan, 1988.

LIANZA S. Medicina de Reabilitação. 3 ed. Rio de Janeiro, Guanabara Koogan, 2001.

MARTINS, D. L.; RABELO, R. J. Influência da atividade física adaptada na qualidade da vida de deficientes físicos. MOVIMENTUM - Revista Digital de Educação Física, Ipatinga: Unileste-MG, v. 3, n. 2, ago/dez. 2008.

MARTINS, J. A.; BARSAGLINI, R. A. Aspectos da identidade na experiência da deficiência física: um olhar socioantropológico. Interface - Comunicação, Saúde e Educação, v. 15, n. 36, p.109-122, 2011.

MATSUMURA, A. D.; RESENDE, J. M.; CHAMLIAN, T. R. Avaliação pré e pós protética da circumetria dos cotos de amputados transtibiais. Acta Fisiátrica, v. 20, n. 4, dez. 2013.

MELO, A. C. R.; LOPEZ, R. F. A. O esporte adaptado. Revista Digital, Buenos Aires, v. 8, n. 51, ago. 2002.

NOCE, F.; SIMIM, A. M.; MELLO, M. T. A percepção de qualidade de vida de pessoas portadoras de deficiência física pode ser influenciada pela prática de atividade física? Rev. Bras. Med. Esporte, v. 15, n. 3, maio/jun., 2009.

OLIVEIRA, A. R. P.; GONÇALVES, A. G.; SEABRA JUNIOR, M. O. Badminton e esporte adaptado para pessoas com deficiência: revisão sistemática da literatura. Revista da Sobama, Marília, v. 18, n. 1, p. 93-108, jan./Jun., 2017. 
PADRÃO, E. C. T. A qualidade de vida dos atletas de bocha paraolímpica de um centro de reabilitação da cidade do Rio de Janeiro. In: DUTRA, F. B.S.; SANTOS, C. R.V.; DI BLASI, F. Aspectos da deficiência: educação, esporte e qualidade de vida. Curitiba: Appris, p. 143$154,2017$.

PEDRINELLI, V. J. Educação física adaptada: conceituação e terminologia. In:

PEDRINELLI, V. J. Educação física e desporto para pessoas portadoras de deficiência. Brasília: MEC/Sedes, p. 7-10, 1994.

RODRIGUES, D. N.; MUSSI, R. F. F.; ALMEIDA, C. B. Atividade física na promoção da saúde de amputados. EFDeportes.com, Buenos Aires, v. 19, n. 191, abr. 2014.

RUFINI, L. H.; TEIXEIRA-ARROYO, C. Atividade física para pessoas com deficiência motora em uma cidade do norte paulista. Revista Educação Física UNIFAFIBE, v. 2, n. 2, p. 99-111, dez., 2013.

SAMULSKI, D. Psicologia do esporte. São Paulo: Manole Ltda, 2002.

SILVA, T. F.; HENRIQUE, A. R. P.; ROCHA, V. L. Acessibilidade: edificações, mobiliários e espaços para uma real inclusão escolar. Rio de Janeiro: Wak, 2015.

VARELA, A. Desporto para as pessoas com deficiência. Revista Educação Especial e Reabilitação, Lisboa, v. 1, n. 5/6, jun., 1989.

WELLICHAN, D. S. P.; SANTOS, M. G. F. Qualidade de vida e deficiência: o CrossFit Adaptado como experiência. 22 f., 2019. [no prelo]

WHEELER, G. D. et al. Personal investmentin disability sport careers: An international study. Adapted Physical Activity Quarterly, v. 16, n. 3, p.219-37, 1999.

\section{Como referenciar este artigo}

WELLICHAN, Danielle da Silva Pinheiro; SANTOS, Marcella Garcia Ferreira dos. Atividade física adaptada para a pessoa com deficiência: o CrossFit Adaptado para um grupo com cadeirantes e amputado. Temas em Educ. e Saúde, Araraquara, v. 15, n. 1, p. 146-158, jan./jun., 2019. e-ISSN 2526-3471. ISSN 1517-7947. DOI: 10.26673/tes.v15i1.12700

Submetido em: 30/11/2018

Aprovado em: 28/02/2019 